Arch. Tierz., Dummerstorf 44 (2001) 3, 329-337

Aus dem Institut fur Emathrungswissenschaften der Martin-Luther-Universitat Halle-Wittenberg

KERSTIN OLOFFS, HEINZ JEROCH UNA PROBEN SORENSEN

\title{
Einsatz eines Nicht-Stărke-Polysaccharid (NSP) hydrolysierenden Enzyms als Futtersupplement bei Legehennen
}

\begin{abstract}
Summary
Title of the paper: Use of a non-starch-polysaccharide (NSP) hydrolysing enzyme as feed supplement for laying hens

The present study investigates the use of a non-starch-polysaccharide (NSP) hydrolysing enzyme as feed supplement for laying hens. Furthermore it should be tested in which extent a multi enzyme complex at two diets ( $24 \%$ barley, $23 \%$ rye, $23 \%$ sunflowerseeds vers. $19 \%$ wheat, $25 \%$ rye, $20 \%$ sunflowerseeds) with two dosages can influence performance parameters, nutrient digestibility and metabolizable energy at laying hens. The investigations comprised six experimental diets. Besides a performance trial with 60 animals per treatment a baiance study with six animals per tratment were carried out. At the performance trial neither between the experimental diets nor between the enzyme dosages statistically differences could be found at the performance parameters. A positive influence of the enzyme supplementation on the reduction of dirty eggs could be observed in both experimental rations; in the course of which the higher enzyme dosage had a bigger effect. At the balance trial no response of the addition of the multi enzyme complex on nutrient digestibility and metabolizable energy could be found.
\end{abstract}

Key Words: Jaying hens, non-starch-polysaccharide (NSP) hydolysing enzymes, performance parameters, nutrient digestibility, dirty eggs

\section{Zusammenfassung}

Im. vorfiegenden Beitrag wird aber Untersuchungen zum Einfluss eines Nicht-Stärke-Polysaccharid (NSP) hydrolysierenden Enzyms als Futtersupplement bei Legehennen berichtet. Es war nachzuweisen, in welchem Umfang ein Multi-Enzym-Komplex bei zwei verschiedenen Rationstypen ( $24 \%$ Gerste, $23 \%$ Roggen, $23 \%$ Sonnenblumensamen bzw. $19 \%$ Weizen, $25 \%$ Roggen, $20 \%$ Sonnenblumensamen) und zwei Dosierungsstufen Einfluß auf die Leistungsparameter, Nahrstoffverdaulichkeit und Umsetzbarkeit der Bruttoenergie bei Legehennen hat, Die Untersuchungen umfassten sechs Futterungsvarianten. Neben einem Leistungsversuch mit jeweils 60 Tieren je Variante wurde ein Bilanzversuch mit jeweils sechs Tieren je Variante durchgefuhrt. Im Leistungsversuch konnten weder zwischen den Rationstypen noch zwischen den unterschiedlichen Enzymdosierungen statistisch signifikante Unterschiede hinsichtlich der Leistungsparameter ermittelt werden. Durch die Enzymsupplementierung konnte bei beiden Rationstypen im Vergleich zur Kontrollgruppe ein Ruckgang der Schmutzeier ermittelt werden, wobei der höheren Enzymdosierung eine gīoßjere Wirkung zuzuschreiben war. Im Bilanzversuch wurde keine Reaktion auf den Zusatz des Multi-Enzym-Präparates bei der Nahrstoffverdaulichkeit and Umsezzbaren Energie festgestellt.

Schlüsseiwörter: Legehennen, Nicht-Starke-Polysacharide (NSP) hydrolysierende Enzyme, Leistungsparameter, Năhrstoffverdaulichkeit, Schmutzeier

1. Einleitung

Die antinutritive Wirkung von Nicht-Stärke-Polysacchariden (NSP) hängt von ihrer chemischen Struktur und Konzentration in den Futterkomponenten ab. Der Futterwert verschiedener Komponenten kann verbessert werden, indem dem Futter NSP-spaltende Enzyme zugesetzt werden. So bekommen NSP-spaltende Enzyme in der Geflügelfutterung eine zunehmende Rolle. Insbesondere bei Broilern konnte bei Rationen mit hohem Anteil an hochpolymeren Nicht-Stärke-Polysacchariden nachgewiesen 
werden, dass durch die erhöhte Verwertung der Futterenergie die Leistungsmerkmale verbessert wurden (JEROCH, 1993; SIMON et al., 1994; DUSEL et al., 1998). Bei Legehennen ist die Wirkung von NSP-spaltenden auf die Leistungsmerkmale und Nährstoffverwertung nach wie vor kontrovers, wobei sich bei Durchsicht der jüngeren Literatur im Vergleich zum Broiler eher ein "Nichtwirken" bezüglich der Leistungsparameter herauskristallisiert (LATTEMANN et al., 1998; OLOFFS et al., 1997, 2000b). Positiv wirkt sich demgegenüber die Enzymsupplementierung auf den Anteil an Schmutzeiern aus (FRAPIN et al., 1997; OLOFFS et al., 1997).

in der vorliegenden Arbeit sollte der Frage nachgegangen werden, ob ein Multi-Enzym-Präparat in zwei Dosierungsabstufungen zu Gerste/Roggen/Sonnenblumen- bzw. Weizen/Roggen/Sonnenblumen-Rationen Auswirkungen auf die Leistungsmerkmale und die Umseizbarkeit der Bruttoenergie bei der Legehenne hat.

2.

Material und Methode

2.1

Leistungsversuch

Der Leistungsversuch wurde mit sechs Gruppen a 60 Tiere (Braune Lohmann Leghorn Hybriden) tuber 12 Wochen durchgeführt. $\mathrm{Zu}$ Versuchsbeginn waren die Legehennen 24 Lebenswochen alt. Die Haltung der Tiere erfolgte in einer Dreietagen-Legehennenbatterie, wobei die Tiere in Einzelkăfigen untergebracht und gleichmäßig im Stall verteilt waren und zu gleichen Anteilen die Etagen belegten. Das Lichtregime wurde nach dem empfohlenen Managementprogramm der Fa. Lohmann fur Braune Legehybriden gesteuert. Wasser und Futter stand den Hennen ad libitum zur Verfigung.

Folgende Merkmale wurden erfasst:

$\begin{array}{ll}\text { * Futterverzehr } & \begin{array}{l}\text { durch laufende Einwaage und Rückwaage der Futterreste } \\ \text { im Abstand von zwei Tagen }\end{array} \\ \text { - Eizahl und Eigewicht } & \text { täglich } \\ \text { - Lebendmasse } & \text { Einzeltierwăgung zu Versuchsbeginn und -ende } \\ \text { - Schmutzeier } & \text { täglich }\end{array}$

\subsection{Bilanzversuch}

36 Braune Lohmann Leghorn Hybriden wurden auf sechs Versuchsgruppen a' sechs Legehennen in Einzelkäfige einer Stoffwechselbatterie aufgeteilt. Die Versuchsperiode setzte sich aus sieben Tagen Vor- und funf Tagen Hauptperiode zusammen. Die Hauptperiode fiel dabei in die 29. Lebenswoche der Hennen. Bis zu Versuchsbeginn bekamen die Hennen ein praxisübliches Legehennenfutter verabreicht, das dann allmählich mit dem Versuchsfutter verschnitten wurde. In der Vorperiode wurde die Futtermenge für die Hauptperiode ermittelt; und diese betrug $86 \mathrm{~g}$ Futter je Legehenne und Tag. Die Tiere wurden zweimal täglich, jeweils vor der Exkrementsammlung, gerüttert. Wasser stand aus Flaschentränken ad libitum zur Verfügung.

Nachfolgende Versuchskriterien wurden in der Bilanzperiode erfasst:

- Eizahl, Eigewicht, Schmutzeieranteil (täglich)

- Lebendmasse zu Beginn und Ende der Hauptperiode 
- Wasserverbrauch (zweimal täglich)

- Exkrementsammlung zweimal täglich, anschließendes Eingefrieren bei $-18^{\circ} \mathrm{C}$

Der Mischplan für die sechs Futtermischungen war zweifaktoriell angelegt (zwei Rationstypen x 3 Zulagestufen). Die Versuchsfuttermischungen für alle Gruppen wurden gleichzeitig und aus identischen Chargen der Einzelkomponenten hergestellt. Rationstyp I enthielt $24 \%$ Gerste, $23 \%$ Roggen und $23 \%$ Sonnenblumen, Rationstyp II $19 \%$ Weizen, $25 \%$ Roggen und $20 \%$ Sonnenblumen. Tabelle 3 zeigt die analysierten Gehalte an Pentosanen und B-Glucan der verwendeten sortenreinen Getreidearten und der Sonnenblumen.

Tabelle 1

Pentosangehait der verwendeten Futterkomponenten ( $\mathrm{g} \mathrm{kg} \mathrm{T}$ ) (Content of pentosanes of the used feedstuffs ( $\mathrm{g} / \mathrm{kg}$ DM))

\begin{tabular}{lccc}
\hline Futterkomponente & Gesamt-Pentosane & Iosliche Pentosane & B-Glucane \\
\hline Gerste "Grete" & 80,2 & 5,5 & 39,1 \\
Roggen "Rapid" & 95,8 & 45,5 & 23,3 \\
Weizen "Zentos" & 59,4 & 7,8 & 5,5 \\
Sonnenblumensamen & 57,2 & 9,1 & n.b. \\
\hline a.b. $=$ nicht bestimmt & & &
\end{tabular}

Beiden Rationen wurde ein Multi-Enzym-Komplex in zwei Dosierungsstufen (500 und 1000 ppm) beigemischt. Grindazym ${ }^{T M}$ GP 5000 (Danisco Ingredients) ist ein staubfreies, grau-weißes Granulat und wird durch die Fermentation eines selektierten Pilzstammes produziert. Der Multi-Enzym-Komplex ist standardisiert auf 12.000 Xylanase Einheiten/g, 5.000 ß-Glucanase Einheiten/g sowie 10 Einheiten Pectinase/g, Zusammengesetzt ist das Enzympräparat aus Protein (Enzym), Fett (Umhüllung) und Weizenstärke (Enzymträger).

Tabelle 2

Zusammensetzung (\%) und inhaltsstoffe (\% bzw. MJ/kg) der verwendeten Futtermischungen (Composition (\%) and ingredients (\% bzw. MJ $/ \mathrm{kg}$ ) of the test diets)

\begin{tabular}{|c|c|c|}
\hline $\begin{array}{l}\text { Komponenten } \\
\text { bzw. Gehalte }\end{array}$ & $\begin{array}{c}\text { Rationstyp I } \\
\text { Gruppe D1, D2, D3 }\end{array}$ & $\begin{array}{c}\text { Rationstyp II } \\
\text { Gruppe D4, DS, D6 } \\
\end{array}$ \\
\hline Gerste & 23,8 & - \\
\hline Roggen & 22,8 & 25,0 \\
\hline Weizen & - & 19,3 \\
\hline Sonnenblume & 23,0 & 20,1 \\
\hline Sojaextraktionsschrot (HP 44) & 17,7 & 19,2 \\
\hline Weizenstärke & - & 5,0 \\
\hline Calciumcarbonat & 8,1 & 8,1 \\
\hline Tierfett & 2,5 & 1,2 \\
\hline DCP & 1,0 & 1,0 \\
\hline DL-Methionin & 0,04 & 0,05 \\
\hline Prămix ${ }^{1}$ & 0,1 & 0,1 \\
\hline AME MJ $/ \mathrm{kg}^{2}$ & 11,17 & 11,18 \\
\hline Rohprotein ${ }^{3}$ & $18,5 / 18,4 / 18,9$ & $18,7 /[8,9 / 19,0$ \\
\hline Rohfeit ${ }^{3}$ & $13,1 / 13,9 / 13,2$ & $9,1 / 9,5 / 9,8$ \\
\hline Lysin' & 0,82 & 0,83 \\
\hline \multicolumn{3}{|c|}{ 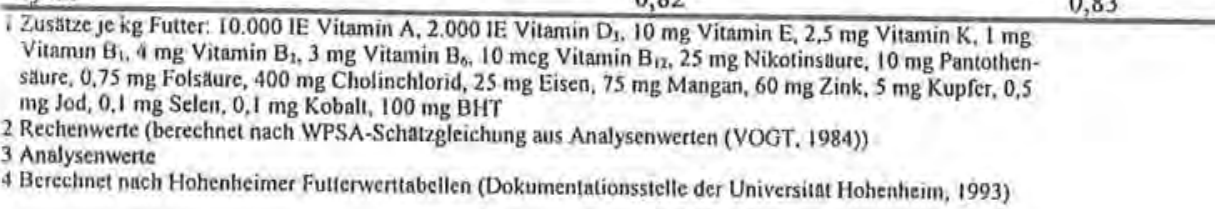 } \\
\hline
\end{tabular}


Zusammensetzung und Inhaltsstoffe der verwendeten Futtermischungen sind aus Tabelle 2 ersichtlich. Die Rationen wurden isocalorisch und isonitrogen hergestellt. Das Futter wurde den Legehennen unpelletiert in Mehlform verabreicht.

Die Analytik der Rohnährstoffe in den Futtermischungen und den Exkrementen wurde nach den Methoden des VDLUFA (1976/1993) vorgenommen. Die Pentosanbestimmung erfolgte nach der Methode von ENGLYST und CUMMINGS (1984), die B-Glucan-Bestimmung nach MCCLEARY und GLENNIE-HOLMES (1985). Die für die Stoffwechseluntersuchungen erforderlichen Bestimmungen des Bruttoenergiegehaltes in den Futtermischungen und Exkrementen wurden mittels adiabatischem Bombenkalorimeter (Typ: IKA-Kalorimeter C 7000) durchgeführt. Um die Umsetzbarkeit der Bruttoenergie zu erhalten, wurde von der mit dem Futter aufgenommenen Energie diejenige in den Exkrementen abgezogen.

Eine firmeninterne Methode (Danisco) zur Ermittlung der Enzymaktivität ergab, dass in den Versuchsrationen die erwartete Enzymaktivität aufgrund der jeweils supplementierten Enzymmenge in allen Fällen 80 \% uberschritt.

Die statistische Auswertung der Versuchsergebnisse erfolgte mit dem Programm für WindowsTM (STATSOFTINC.,1995). Die Ergebnisse werden in den Tabellen als arithmetische Mittelwerte mit den dazugehörigen Standardabweichungen angegeben. Bei allen Vergleichen wurde von einer Irrtumswahrscheinlichkeit von $5 \%$ ausgegangen $(p<0,05)$. Zur Kennzeichnung von Signifikanzen zwischen den Varianten in den Tabellen bedeuten gleiche Buchstaben, dass keine statistisch gesicherten Unterschiede bestanden.

\section{Ergebrisse \\ 3.1 Leistungsversuch}

Der Versuch lief störungsfrei ab. Über alle Gruppen wurde eine Verlustrate von 2,5\% beobachtet, wobei die Tierverluste unabhängig von den Fütterungsvarianten waren. In Gruppe D1 wurde ein Nichtleger festgestellt, der bei der statistischen Auswertung nicht berücksichtigt wurde.

In Tabelle 3 sind die ermittelten Leistungskriterien des Leistungsversuches aufgeführt. Der durchschnittliche tăgliche Futterverzehr aller Versuchsgruppen betrug 111,6 g. Zwischen den Versuchsgruppen ergaben sich keine Unterschiede. Weder zwischen den Rationstypen noch zwischen den unterschiedlichen Enzymdosierungsstufen konnten bei den erhobenen Leistungsmerkmalen statistisch signifikante Unterschiede ermittelt werden. Beide Kontrollgruppen (D1 und D4) wiesen ein hohes Leistungsniveau auf. So betrug die durchschnittliche Legeintensität bei den mit Gerste/Roggen gefütterten Legehennen $95 \%$, die Hennen, die die Ration mit Weizen/Roggen erhielten, erreichten eine Legeintensität von $96 \%$.

Ein Einfluß der Enzymsupplementierung ließ sich beim Schmutzeieranteil feststellen. Durch die Enzymergänzung konnte bei beiden Rationstypen im Vergleich zur Kontrollgruppe ein Rückgang der Schmutzeier ermittelt werden. Desweiteren war die höhere Dosierung des Enzymprăparates (1000 ppm) bezüglich Schmutzeiersenkung der geringeren Enzymgabe (500 ppm) uberlegen. Die Rationen mit Weizen/Roggen wiesen im Vergleich zu den Rationen mit Gerste/Roggen insgesamt eine geringere Anzahl an Schmutzeiern auf. 
Über den gesamten Versuchszeitraum von 12 Wochen zeigten die Hennen aller Versuchsgruppen eine positive Gewichtsentwicklung auf, die auf einheitlichem Niveau lag. Die Hennen wiesen zu Versuchsbeginn ein mittleres Gewicht von $1720 \mathrm{~g}$ auf.

Tabelle 3

Leistungsdaten Uber 12 Versuchswochen (Performance data over 12 trial weeks)

\begin{tabular}{|c|c|c|c|c|c|c|c|c|c|c|}
\hline $\begin{array}{c}\text { Grup- } \\
\text { pe }\end{array}$ & $\begin{array}{l}\text { Ra- } \\
\text { tions- } \\
\text { typ }\end{array}$ & $n$ & $\begin{array}{c}\text { Enzym- } \\
\text { zusaiz }\end{array}$ & $\begin{array}{c}\text { Futter- } \\
\text { verzehr } \\
\text { g/Henne } \\
\text { Tag }\end{array}$ & $\begin{array}{c}\text { Legein- } \\
\text { tensitat } \\
\%\end{array}$ & $\begin{array}{c}\text { Einzel- } \\
\text { eimasse } \\
\mathrm{g}\end{array}$ & $\begin{array}{c}\text { Tägl. } \\
\text { Eimasse } \\
/ \\
\text { Henne } \\
\text { g }\end{array}$ & $\begin{array}{l}\text { Futterauf- } \\
\text { wand } \\
\mathrm{kg} \text { Futter/ } \\
\mathrm{kg} \mathrm{Ei-} \\
\text { masse }\end{array}$ & $\begin{array}{l}\text { Lebend- } \\
\text { masse- } \\
\text { ver- } \\
\text { anderung } \\
\text { in g/Tier } \\
\text { 1.-84. Tag }\end{array}$ & $\begin{array}{c}\text { Schmutz- } \\
\text { eier- } \\
\text { anteil } \\
\%\end{array}$ \\
\hline DI & $\mathrm{G} / \mathrm{R}$ & 57 & $\cdot$ & 112 & 95 & 58 & $\begin{array}{c}56 \\
4\end{array}$ & 1,986 & +177 & 5,9 \\
\hline D2 & $\mathrm{G} / \mathrm{R}$ & 59 & $500 \mathrm{ppm}$ & $\begin{array}{c}8 \\
109\end{array}$ & $\begin{array}{c}4 \\
94\end{array}$ & $\begin{array}{c}3 \\
59 \\
4\end{array}$ & $\begin{array}{c}4 \\
56 \\
4\end{array}$ & $\begin{array}{l}0,162 \\
1,910 \\
0,150\end{array}$ & $\begin{array}{c}102 \\
+138 \\
120\end{array}$ & 5,4 \\
\hline D4 & W/R & 59 & . & $\begin{array}{c}8 \\
114 \\
9\end{array}$ & $\begin{array}{c}5 \\
96 \\
5\end{array}$ & $\begin{array}{c}3 \\
58 \\
5\end{array}$ & $\begin{array}{l}4 \\
56 \\
4\end{array}$ & $\begin{array}{l}0,152 \\
2,004 \\
0,169\end{array}$ & $\begin{array}{c}+169 \\
102\end{array}$ & 5,6 \\
\hline DS & $W / R$ & 58 & $500 \mathrm{ppm}$ & $\begin{array}{c}112 \\
9\end{array}$ & $\begin{array}{c}96 \\
6\end{array}$ & $\begin{array}{c}58 \\
4\end{array}$ & $\begin{array}{c}57 \\
4\end{array}$ & $\begin{array}{l}1,953 \\
0,169\end{array}$ & $\begin{array}{r}+185 \\
145\end{array}$ & 5,3 \\
\hline D6 & W/R & 59 & $1000 \mathrm{ppm}$ & $\begin{array}{l}111 \\
11\end{array}$ & $\begin{array}{c}96 \\
4\end{array}$ & $\begin{array}{c}58 \\
4\end{array}$ & 56 & $\begin{array}{l}2,019 \\
0,166\end{array}$ & $\begin{array}{c}+197 \\
118\end{array}$ & 4,2 \\
\hline
\end{tabular}

$\mathrm{G} / \mathrm{R}=\mathrm{Gerste} / \mathrm{Roggen} W / \mathrm{R}=$ Weizen/Roggen

\subsection{Bilanzversuch}

Die insgesamt 36 Legehennen wurden mit einer möglichst einheitlichen Lebendmasse in die Stoffwechselkäfige aufgestallt. Das durchschnittliche Einstallungsgewicht betrug $1712 \mathrm{~g}$. Der tägliche Futterverzehr betrug in allen Versuchsgruppen einheitlich 85 g. Hinsichtlich der Leistungsmerkmale (Legeleistung, Einzeleimasse, tägliche Eimasse) konnten in der Bilanzperiode keine Unterschiede ermittelt werden. Weder der Rationstyp noch die Enzymdosierung hatten in diesem Fall Auswirkungen auf die

Tabelle 4 Nahrstoffverdaulichkeit und Umsetzbarkeit der Bruttoenergie im Bilanzversuch (Nutrient digestibility and
metabolizable energy in the balance trial)

\begin{tabular}{|c|c|c|c|c|c|c|c|c|}
\hline Gruppe & $\begin{array}{l}\text { Rations- } \\
\text { typ }\end{array}$ & $\mathrm{n}$ & $\begin{array}{c}\text { Enzym- } \\
\text { zusatz }\end{array}$ & $\begin{array}{c}\text { Rohprotein } \\
\text { Umserz- } \\
\text { barkeit } \\
\%\end{array}$ & $\begin{array}{c}\text { Rohfett } \\
\text { Verdau- } \\
\text { lichkeit } \\
\%\end{array}$ & $\begin{array}{c}\text { Umsetzbarkeit } \\
\text { der Brutto- } \\
\text { energie } \\
\%\end{array}$ & $\begin{array}{l}\text { AME } \\
\text { MJ/kg T }\end{array}$ & $\begin{array}{l}\text { Wasser- } \\
\text { verbrauch } \\
\text { g/Henne/ } \\
\text { Tag }\end{array}$ \\
\hline \multirow[t]{2}{*}{ DI } & $\mathrm{G} / \mathrm{R}$ & 6 & - & 47,25 & $88,30^{\pi}$ & 68,16 & 10,90 & 377 \\
\hline & & & & 4,12 & 1,41 & 1,06 & 0,17 & 100 \\
\hline \multirow[t]{2}{*}{ D2 } & $\mathrm{G} / \mathrm{R}$ & 6 & $500 \mathrm{ppm}$ & 41,12 & $80,21^{b}$ & 65,58 & 10,30 & 242 \\
\hline & & & & 3,87 & 8,36 & $4,4 !$ & 0,69 & 134 \\
\hline \multirow[t]{2}{*}{ D3 } & G/R & 6 & $1000 \mathrm{ppm}$ & 46,52 & $83,71^{\mathrm{ab}}$ & 66,01 & 10,23 & 324 \\
\hline & & & & 4,22 & 2,85 & 1,92 & 0,30 & 100 \\
\hline \multirow[t]{2}{*}{ D4 } & W/R & 6 & - & 45,28 & 81,49 & 67,68 & 10,19 & $317^{\text {sb }}$ \\
\hline & & & & 5,18 & 4,54 & 3,63 & 0,55 & 129 \\
\hline \multirow[t]{2}{*}{ D5 } & $W / R$ & 6 & $500 \mathrm{ppm}$ & 43,14 & 76,00 & 65,55 & 9,84 & $357^{\circ}$ \\
\hline & & & & 2,80 & 6,32 & 2,08 & 0,31 & 59 \\
\hline \multirow[t]{2}{*}{ DG } & $W / R$ & 6 & $1000 \mathrm{ppm}$ & 44,06 & 83,21 & 68,93 & 10,34 & $194^{\circ}$ \\
\hline & & & & 6,58 & 3,85 & 4,27 & 0,64 & 54 \\
\hline
\end{tabular}


Leistung der Legehennen. Da es sich um nur einen kurzen Prüfzeitraum handelt, wird auf die Ergebnisdarstellung verzichtet. Schmutzeier konnten während der Prüfperiode bei keiner Versuchsgruppe konstatiert werden. In Tabelle 4 sind die Nährstoffverdaulichkeit bzw. -verwertung sowie die Umsetzbarkeit der Bruttoenergie und der Wasserverbrauch aufgeführt.

Die Umsetzbarkeit des Rohproteins wurde bei beiden Rationstypen nicht durch die Enzymsupplementierung beeinflusst. Der statistisch signifikante Unterschied in der Verdaulichkeit des Rohfettes betraf Gruppe D1 mit 88,3\% und Gruppe D2, die mit 500 ppm ergänzte Enzymgruppe, in der eine statistisch signifikant geringere Rohfettverdaulichkeit von nur $80,1 \%$ erreicht wurde.

Umsetzbarkeit der Bruttoenergie und der AME-Gehalt zeigten in diesem Versuch ebenso keine Reaktion auf den Zusatz eines NSP-spaltenden Enzyms. Auffallend ist, da.3 beide Gruppen mit dem Enzymzusatz von 500 ppm (Gruppe D2 und D5) bei den genannten Merkmalen im Vergleich zur Kontrollgruppe als auch zu der Gruppe mit 1000 ppm fast durchgehend die schlechtesten Versuchsergebnisse aufzuweisen haben, wenn auch keine statistsich signifikanten Unterschiede vorliegen.

Der tägliche Wasserverbrauch lag zwischen minimal $194 \mathrm{~g}$ und maximal $377 \mathrm{~g}$. Bei den Weizen/Roggen-Versuchsdiäten konnte zwischen den enzymergänzten Rationen (D5 und D6) ein signifikanter Unterschied ermittelt werden. Die Gruppe mit der höheren Enzymdosierung (D6) wies bei dieser Rationszusammensetzung den geringsten Wasserverbrauch pro Legehenne und Tag auf.

\section{Diskussion}

Ziel der vorliegenden Arbeit war es, die Enzymergänzung zu Futtermischungen mit erhöhten Gehalten an löslichen Pentosanen, welcher durch Roggenintegration erreicht wurde, zu prüfen. Außerdem sollten die verwendeten sortenreinen Einzelfuttermittel einen hohen Gehalt an B-Glucan vorweisen. Die Gesamt-NSP- und B-Glucan-Gehalte der eingesetzten Getreidearten liegen auf vergleichbarem Niveau zu den Literaturangaben (JUODEIKIENE et al., 1996; DUSEL, 1998; JEROCH et al.,1999). Die kalkulierten Gehalte der als "antinutritiv" bezeichneten NSP zeigten, dass die Gerste/Roggen /Sonnenblumen-Rationen im Vergleich zu den Gruppen mit den Komponenten Weizen/Roggen/Sonnenblume geringfügig höhere Werte aufwiesen.

Wie bereits einige Arbeiten zum Einsatz von NSP-spaltenden Enzymen bei Legehennen unter Verwendung verschiedenster Rationen und unterschiedlicher Versuchsdauer keine Wirkung auf die Leistungsparameter nachweisen konnten (AL BUSTANY und ELWINGER, 1988; GRUZAUSKAS et al., 1991; BENABDELJELIL, 1991,1992; BRENES et al., 1994; BRUFAU et al., 1994; FRANCESCH et al., 1994; HALLE, 1997; OLOFFS et al., 1997, 2000b; LATTEMANN et al., 1998), zeigte der vorgestellte Versuch ebenfalls keinen positiven Einfluß einer Supplementierung von NSPspaltenden Enzymen auf die Leistungsmerkmale bei der Legehenne. Obwohl in den zwei unterschiedlichen Rationstypen $23 \%$ bzw, $25 \%$ Roggen integriert waren, zeigte der hohe tägliche Futterverzehr eine gute Akzeptanz der Versuchsmischungen. Da die Legeintensität im durchgeführten Versuch sehr hoch war, ist eine nachweisliche Wirkung einer Enzymsupplementierung zudem äußerst schwierig.

Eindeutig positive Effekte NSP-spaltender Enzyme im Legehennenbereich ergaben 
sich hinsichtlich der Einstreuqualităt (FRANCESCH et al., 1994) bzw. beim Anteil von Schmutzeiern. Die Enzymergänzung zu verschiedenen Rationszusammensetzungen verdeutlichte, dass sich der Anteil der Schmutzeier reduzierte (FRAPIN et al., 1997; HADORN et al., 1997; OLOFFS et al., 1997, 2000b). Die ermittelten Resultate ordnen sich in die bisherige Ergebnisdarstellung ein. Begründet werden kann diese Tatsache durch eine reduzierte Viskosităt der Exkremente nach Ergänzung von Enzympräparaten, was im vorgestellten Versuch jedoch nicht gepruft wurde.

Der Zusatz des NSP-spaltenden Enzyms hatte im vorgestellten Bilanzversuch keine positiven Auswirkungen auf die Verdaulichkeit der Rohnăhrstoffe und die Umsetzbarkeit der Bruttoenergie. Die faecale Rohproteinumsetzbarkeit und Rohfettverdaulichkeit lagen im Vergleich zu anderen Versuchen (LATTEMANN et al., 1999; OLOFFS et al, 1999,2000 a) auf recht hohem Niveau. Einen statistisch zu sichernden Einfluss des Aiters auf die Verdaulichkeit des Rohfettes (36., 48., 60., 72. Lebenswoche) konnten LATTEMANN et al. (1999) feststellen, wobei die in der 72. Lebenswoche erreichte Rohfettverdaulichkeit mit $62,5 \%$ noch unter dem Minimalwert von $76 \%$ des vorgestellten Versuches liegt. Deutlich geringer als in den oben erwăhnten Literaturangaben ist die Umsetzbarkeit der Bruttoenergie und der AME-Gehalt. Die Weizen-RoggenRationen enthielten weniger Rohfeit, was dazu beiträgt, die niedrigeren Werte dieser Gruppe zu erklären, jedoch keine Erklärung der insgesamt niedrigen Resultate aufzeîgt.

Der tägliche Wasserverbrauch der Legehennen zeigte sich teilweise signifikant durch die Enzymsupplementierung beeinflusst, jedoch lässt sich aus dem vorgestellten Versuchsergebnis keine eindeutige Schlussfolgerung ableiten. BRUFAU et al. (1994) ermittelten den Wasserverbrauch bei Legehennen, die Rationen auf Gerstenbasis ohne und mit Enzymzusatz erhielten. Der Wasserverbrauch der Legehennen war durch die Enzymsupplementierung nicht beeinflusst, aber das Verhältnis von Wasser- zu Futterverbrauch wurde durch die Enzymergänzung signifikant reduziert.

Insgesamt betrachtet, reihen sich der Leistungs- als auch Bilanzversuch in die Versuchsergebnisse ein, in denen der Einsatz eines NSP-spaltenden Enzymes als Futtersupplement bei Legehennen keine positiven Auswirkungen auf die Leistungsparameter aufweist. Im vorgestellten Versuch wurden im Vergleich zu weiteren am gleichen Institut durchgeführten Versuchen (OLOFFS et al,, 1997 und 2000b) andere Getreidesorten und Enzympräparate als auch Dosierungen verwendet. Dennoch konnte auch bei unterschiedlicher Ausgangssituation keine positive Enzymwirkung auf die Leistungsparameter bei adultem Huhnergeflügel konstatiert werden.

\section{Literatur}

AL. BUSTANY, Z; ELWINGER, K.:

Whole grains, unprocessed rapeseed and B-zlucanse in diets for laying hens. Swed. J. Agric. Res. 18 (1988), 3 l- 40

BENABDELJELIL, K.:

Effects of enzyme supplementation of barley based diets on hen performance and egg quality. Proc. $8^{\text {th }}$ European Symposium on Poultry Nutrition, 14-17 Oct., Venezia, Italy, 337-342, 1991

BENABDELJELIL, K.:

Improvement of barley utilization for layers: effects on hen performance and egg quality. Proc World's Poultry Congress, 20-24 Sept., Amsterdam, The Netherlands, 405-409, 1992 
BRENES, A.; GUENTER, W.; MARQUARDT, R.R.; ROTTER, B.A.:

Effect of beta-glucanase/pentosanes enzyme supplementation on the performance of chickens and laying hens fed wheat, barley, naked oats and rye diets. Can. J. Anim. Sci. 73 (1994) 4, 941-951

BRUFAU, J.; COS, R.; PEREZ-VENDRELL, A.; ESTEVE-GARCIA, E.:

Performance of laying hens affected by the supplementation of a barley-based diet with a crude enzyme preparation from Trichiderna viride. Can. J. Anim. Sci. 74 (1994), 129-133

Dokumentationsstelle der Universitat Hohenheim:

Năhrsioff-, Mineralstoff- und Aminosăuretabellen zur Geflugelfutterung. In: Jahrbuch fur die GefluDUSEL, G. gelwirtschaft 1997, Hrsg. J. PETERSEN, Verlag Eugen Ulmer (1997)

Untersuchungen zur Variabilitat des Futterwertes von Weizen unter besonderer Berucksichtigung der Arabinoxylane sowie zur Wirksamkeit von Nicht-Starke-Polysaccharid (NSP)-hydrolysierenden Enzymen in weizenbetonten Rationen beim Broiler und Ferkel. Univ. Halle-Wittenberg, Diss., 1998

DUSEL, G.; KLUGE, H.; JEROCH, H.; SIMON, O.:

Xyisrase supplementation of wheat-based rations for broilers: influence of wheat characteristics. $J$. Appl. Poutiry Res, 7 (1998), 119-131

ENGLYST, H.N.; CUMMINGS, J.H.:

Simplified method for the measurement of total non-starch polysaccharides by gas-liquid chromatography of constituent sugars as alditol acetates, Analyst 109 (1984), 937-942

FRANCESCH. M.; PEREZ-VENDRELL, A.; ESTEVE-GARCIA, E;: BRUFAU, $y_{\text {: }}$

Effects of enzyme supplementation of a barley and sunflower based diet on laying hen performance. Proc. $9^{\text {th }}$ WPSA Poultry Conf, Glasgow, UK, 425-426, 1994

FRAPIN, D.; GERAERT, P.A.; UZU, G.:

NSP-enzyme improves feed digestibility and performance in adult hens fed wheat based diet. Proc, $11^{\text {th }}$ European Symposium on Poultry Nutrition, Faaborg, Denmark, 481-483, 1997

GRUZAUSKAS, R.; JEROCH, H.; JÄNISCH, S.; GIRING, H.; JEROCH, K.:

Enzymerganze Futtermischungen auf Gerstebasis fur Legehennen. Proc. Int. Scientific Conf, 14-16 May, Kaunas, Litauen, 215-218, 1991

HADORN, R; GLOOR, A.; WIEDMER, H.:

Efrect of a carbohydrase in an energy-reduced and wheat based diet for laying hens. Archiv fur Geflugelkunde 61 (1997) 2, 82-87

HALLE, I.:

Untersuchungen zur Wirksamkeit von NSP-spaltenden Enzymen bei Legenennen und unterschiedtichem Energiegehalt des Futters. Vortrag 1. Domburger Fachgespräch, Dornburg (Saale), 23.09.1997

JEROCH, $\mathrm{H}$ :

Zum Einsatz von Nicht-Starke-Polysaccharide spaltenden Enzymen in der Geflugelernathrung. Proc. $4^{\text {th }}$ Symp. Vitamine und Zusatzstoffe bei Mensch und Tier, Jena, 342-353, 1993

JEROCH, H.; KLUGE, H.; SIMON, O.; LENGERKEN, J. V.:

Inhaltsstoffe und Futterwertdaten von Getreide und Kornererbsen. Hrsg.: Institut fur Ernfhrungswissenschaften der Martin-Luther-Universitat Halle-Wittenberg. ISBN 3-86010-543-4, 1999

JUODEIKIENE, G.; GRUZAUSKAS, R.; SESKEVICIENE; J.; CECENIKOVAITE, A.; DEGUTYTE, L.; JEROCH, H..

Nahrstoffgehalze verschiedener in Litauen angebauter Getreidearten. 4. Tagung Schweine- und Geflugelernahrung, Halle/Saale, 117-123, 1996

MCCLEARY, B.Y.: GLENNIE-HOLMES, M.:

Enzymatic quantification of (1-3)-B-D-Glucan in barley and malt. J. Inst. Brew 91 (1985), 285-295

LATTEMANN, D.; HALLE, I; RAUCH, H.-W.; FLACHOWSKY, G.:

Zusatzstoffe in der Legehennenfutterung: Zur Wirksamkeit einer Xylanase und eines Antibiotikums bei alleiniger oder kombinierter Anwendung in der Legehennenfutterung, VDLUFA, Gießen 1998, $405-408$

LATTEMANN, D,; HALLE, I.; RAUCH, H.-W.; FLACHOWSKY, G.:

Untersuchungen zum Einflußs einer Xylanase und eines Antibiotikums auf die Verdaulichkeit von Rohnathrstoffen und NSP-Fraktionen im Digestionstrakt von Legehennen. VDLUFA, Halle/Saale 1999, 147

OLOFFS, K, JEROCH, H.; SCHÖNER, F,-J.:

Zur Wirksamkeit von Nicht-Starke-Polysaccharide (NSP) hydrolysierenden Enzymen als Futtersupplemente zu Legehennenrationen auf Gerste-Roggen- und Weizen-Roggen-Basis. J. Anim. Physiol a. Anim. Nutr. 78 (1997), 178-186

OLOFFS, K; JEROCH, H.; SCHONER, F,-J..

Zur Wirksamkeit Nicht-Starke-Polysaccharide hydrolysierender Enzyme auf die Nahrstoffverdaulichkeit und Umsetzbarkeit der Bruttoenergie von Rationen auf Gerste-Roggen- und Weizen-Roggen-Basis bei Legehennen. Arch. Anim. Nutr, 52 (1999), 155-165 
OLOFFS, K.; SAMLI, E.; JEROCH, H.:

Untersuchungen zum Einfluß einer Xylanaseergänzung in Legehennenrationen auf Weizenbasis. I.

Mith.: Auswirkungen auf die praecanale und fanale Nahrstoffverdaulichkeit sowie umsetzbare Energic.
J. Anim. Physiol. a. Anim. Nutrition 84 (2000), 125-135

OLOFFS, K.; SAMLI, B.; JEROCH, H.:

Untersuchungen zum Einfluß eỉner Xylanaseergänzung in Legehennenrationen auf Weizenbasis. 2

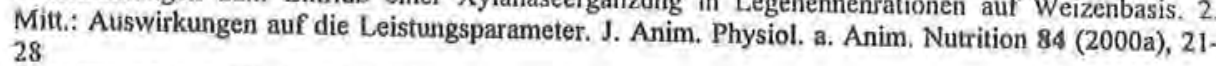

SIMON, O.; RUTTLOFF, H,; KLAPPACH, G.:

Einsatz von Enzymprtaparaten in der Tieremăhnung und in der Futtermittelindustrie. In: Industrielle EnSTATSOFTINC.

VDLUFA

Tulsa OK: statistica for windows ${ }^{\mathrm{TM}}$ operating system (Realease 5.0), 1995

Verband Deutscher Landwirtschafticher Untersuchungs- und Forschungsanstalten. Methodenbuch: Die chemische Untersuchung von Futtermitteln. 3. Erganzungslieferung 1993, VDLUFA-Veriag, Darmstadt,
$1976 / 1993$ VOGT, $\mathrm{H}_{\text {: }}$ :

Die neue WPSA-Formel zur Schätzung des Gehaltes an umsetzbarer Energie in Geflugelmischfutter.
Kraffutter 67 (1984), 274

Eingegangen: 12.01 .2000

Akzeptiert: 14.12 .2000

Anschrin der Verfasser

Dr. KERSTIN OLOFFS, Prof, Dr. HEINZ JEROCH, PROBEN SORENSEN

Institut fur Ernăhrungswissenschaften der

Martin-Luther-Universitat Halle-Wittenberg

Emil-Abderhaldenstr. 26

D-06108 Halle 
Arch. Tierz, Dummerstorf 44 (2001) 3, 338

\title{
Buchbesprechung
}

\section{Pareys Lexikon der Syndrome - Erb- und Zuchtkrankheiten der Haus- und Nutztiere}

\author{
ALEXANDER HERZOG
}

S16 Seiten, 136 Abbildungen, 63 Tabellen, Parey Buchverlag Berlin, im Blackwell Wissenschaftsverlag, 2001 ISBN 3-8263-3237-7, DM 198,00; EUR 101,24; oS 1445,00; sFr 174,00

Die veterinärmedizinische Genetik hat in den letzten Jahrzehnten bedeutende Fortschritte erzielt. Neben den kleineren Haustieren, gewinnen bei den landwirtschaftlichen Nutztieren unter intensiven Zuchtungsmaßnahmen, modernen Biotechniken, wie kunstlicher Besamung, Embryotransfer u,a, die heterogene Gruppe von Erb- und Zuchtkrankheiten besonders an Bedeutung. Dank der sich standig entwickeinden Diagnostikverfahren, hat sich die Erkennbarkeit dieser Krankheiten verbessert. Als Ursachen dieser Krankheitsgruppe werden Genmutationen, numerische und strukturelle Chromosomenaberrationen angesehen, Auch selbst nicht genetisch verursachte Krankheitssymptome können durch genetisch bedingte Konstitution und Leistungsveranlagung begunstigt werden. Den Tangens zwischen Veterinărmedizin und Zuchtung bei dieser Krankheitsgruppe aufzuhellen, den Krankheitswert und die Behandlungschancen in aberschaubarer, handhabbarer Form zu vermitteln, ist mit diesem Buch ein dankenswertes Verdienst des Autors. Dies um so mehr, als im Rahmen des veterinarmedizinischen Studiums kein klinisch-genetisches Grundwissen mehr vermittelt wird und das vorliegende Buch deshalb besonders hilfreich sein kann.

Die im Buch getroffene Auswahl bericksichtigt die wichtigsten und häufigsten Krankheitsbilder, wobei sehr selten auftretende oder solche an deren Zustandekommen genetischen Faktoren eine nur untergeordnete Rolle spieien, nicht einbezogen werden. Begriffe aus der Kleintierpraxis dominieren. Das ist erklarbar aus der Tatsache, dass bei landwirtschaftichen Nutztieren seit Jahrhunderten eine staatliche Zuchttierlberwachung, später Zuchtdokumentation vorliegt, die erkennbare Erbkrankheiten für den Tierarzt und den Zuchter-kontrollierbarer machte.

Samtliche Krankheitsbilder werden nach den gebräuchlichen Benennungen lexikongerecht, alphabetisch geordnet. Unter anderen Synonymen wird jeweils auf die entsprechende Darstellung, die abhängig von der Bedeutung und dem Wissensstand unterschiedlich umfangreich ist, hingewiesen. Es finden sich, wo erforderlich, zu den einzelnen Begriffen die Informationen uber Definition, Vorkommen, Krankheitswert, Genetik, Therapie, Maßrahmen und Literatur bzw, die Besonderheiten, die fur die jeweilige betroffene Tierart beschrieben werden. Zahlreiche Abbildungen und Tabellen erhohen den Informationswer des Buches, wenngleich es bei ailer Informationsfulle nicht die Absicht des Autors war, spezielle Lehr- und Fachbücher zu ersetzen. Das vorliegende Buch isi tinter den tiermedizinischen Pathogenetikbachern das derzeit umfassendste deutschsprachige Werk zu Fehlbildungen. Sowohl fur die Aus- und Weiterbildung als auch für den praktischen Tierarzt und Tierzllchter ermöglicht es, neben der Information uber Erblichkeit und Erbgang einer Krankheit, auch eine Orientienung uber den zu enwartenden Krankheitswert und damit zusammenhängende Behandlungschancen sowie uber mögliche oder notwendige genetische Spezialuntersuchungen und-maßnahmen. Der Nutzer dieses Nachschlagewerkes erhält fưr eine Fülle von Fragen aus der Praxis Antworten, die auf fundiertem, neuestem, tiermedizinischem und tierzllchterischem Fachwissen beruhen. Dieses Buch ist ein unentbehrliches Hilfsmittel nicht nur fur Studierende der Veterinärmedizin und Landwirtschaft oder Tierárzte, sondem auch für Zuchter und in der landwirtschaftlichen Beratung Tatige. 